\title{
Dynamic Characteristics Analysis of a Multi-scroll Conservative Chaotic System with Sinusoidal Nonlinearity
}

\author{
Zhonggao Chen, Minghan Song \\ Department of Automation, Tianjin University of Science and Technology, \\ 1038 Dagunanlu Road, Hexi District, Tianjin 300222, PR China \\ E-mail:*313263747@qq.com
}

\begin{abstract}
In this paper, a multi-scroll conservative chaotic system with sinusoidal nonlinearity is studied. Based on theoretical analysis and numerical analysis, such as equilibrium point and its stability analysis, Lyapunov exponent spectrum and bifurcation diagram, the system is found to show some complex dynamics. In addition, NIST test also verify that the pseudo randomness of the proposed system is satisfactory.
\end{abstract}

Keywords: multi-scroll, conservative, NIST test

\section{Introduction}

In 1993 , Suykens ${ }^{1}$ first proposed a method to generate $n$ double scroll attractors by adding breakpoints. Compared with single scroll chaotic system or double scroll chaotic system, multi-scroll chaotic system has more control parameters and corresponding key parameters. In addition, it can present complex multi-directional grid like vortices in phase space. The number and shape of vortices can also be controlled and adjusted by the parameters of the system. It has more complex dynamic behavior and is more conducive to image encryption. Therefore, multi-scroll chaotic systems have attracted many attentions, and different types of multi-scroll chaotic systems have been reported, such as unidirectional (1D) multi-scroll chaotic systems ${ }^{2,3}$, bidirectional (2D) multi-scroll chaotic systems $^{4-8}$, three-dimensional (3D) multi scroll chaotic systems $^{9}$, and multi-directional multi-scroll chaotic systems ${ }^{10-12}$. In addition, the realization of chaotic system based on FPGA is also studied ${ }^{13,14}$. In three-dimensional differential equations, the general jerk system has fewer terms, so chaotic systems based on general jerk system have been widely studied ${ }^{15-19}$. In practical application, the increase of vortex number in chaotic system is realized by increasing the number of equilibrium points. Due to the periodicity of sinusoidal functions, many researchers design multi-scroll chaotic systems by modifying the expression of sinusoidal functions or adding control functions to chaotic systems with sinusoidal functions ${ }^{15,16,18,20-22}$.

However, there are few studies on the multi-scroll attractors of conservative chaotic systems. In this paper, a new conservative chaotic system is constructed by using sinusoidal function. Through Lyapunov exponent diagram, bifurcation diagram and phase diagram, it is found that there are different attractors with different scroll numbers in the case of different initial values.

\section{Construction of Four-dimension Conservative System}

Firstly, a new four-dimension conservative system is constructed. It can be expressed as:

$$
\dot{\mathbf{x}}=J(\mathbf{x}) \nabla H(\mathbf{x})\left[\begin{array}{cccc}
0 & a & 0 & b y \\
-a & 0 & 0 & 0 \\
0 & 0 & 0 & c \\
-b y & 0 & -c & 0
\end{array}\right]\left[\begin{array}{c}
x \\
y \\
z \\
W
\end{array}\right]
$$

(C) The 2021 International Conference on Artificial Life and Robotics (ICAROB2021), January 21 to 24, 2021 
Where, $J(\mathbf{x})=\left[\begin{array}{cccc}0 & a & 0 & b y \\ -a & 0 & 0 & 0 \\ 0 & 0 & 0 & c \\ -b y & 0 & -c & 0\end{array}\right], \nabla H(\mathbf{x})=\left[\begin{array}{c}x \\ y \\ z \\ w\end{array}\right]$.

Secondly, set $(a, b, c)=(4,4,4)$, equation (1) can be described as:

$$
\left\{\begin{array}{l}
\dot{x}=4 y+4 y w \\
\dot{y}=-4 x \\
\dot{z}=4 w \\
\dot{w}=-4 x y-4 z
\end{array}\right.
$$

Where $\mathrm{x}, \mathrm{y}, \mathrm{z}$ and $\mathrm{w}$ is the state variable, and $\mathrm{a}, \mathrm{b}, \mathrm{c}$ is system parameter, respectively.

\subsection{Conservative characteristics of system (2)}

The divergence of the system (2) is

$$
\nabla f=\frac{\partial \dot{x}}{\partial x}+\frac{\partial \dot{y}}{\partial y}+\frac{\partial \dot{z}}{\partial z}+\frac{\partial \dot{w}}{\partial w}=0
$$

Moreover, the time domain derivative of the energy function is

$$
\dot{H}=\nabla H(\mathbf{x})^{T} J(\mathbf{x}) \nabla H(\mathbf{x})=0
$$

According to the above analysis, the system satisfies both Hamiltonian energy conservation and volume conservation.

\subsection{Equilibrium point of the system (2)}

The equilibrium point of the system (2) is obtained as $(0,0,0,0)$, and the corresponding eigenvalue is $(6 i,-6 i, 6 i,-6 i)$. Set $\nabla H=(\sin (x), y, z, w)$, an infinite number of equilibrium points are obtained. And equation (2) can be express as:

$$
\left\{\begin{array}{l}
\dot{x}=4 y+4 y w \\
\dot{y}=-4 \sin (x) \\
\dot{z}=4 w \\
\dot{w}=-4 \sin (x) y-4 z
\end{array}\right.
$$

It can be found that the equilibrium point of the system (5) is $(k \pi, 0,0,0), k \in Z^{*}$. When the equilibrium point meets $(2 k \pi, 0,0,0)$, the corresponding eigenvalue is $(4 i,-4 i, 4 i,-4 i)$, and the equilibrium point type is a center point; when the equilibrium point is $((2 k-$ 1) $\pi, 0,0,0)$, the corresponding eigenvalue is $(4,-4,4 i,-4 i)$, and the type of the equilibrium point is an unstable saddle point.

\section{The Dynamic Characters of the System (5)}

In this part, the dynamic characteristics of the system (5) are analyzed through Lyapunov exponent spectrum and phase diagram .

\subsection{Lyapunov exponent spectrum of the system (5)}

Set the initial value $(x, y, z, w)=(x(0), 1,1,1)$, Lyapunov exponent diagram of the system (5) with the change of the initial $x(0)$ is shown in Fig. 1 .

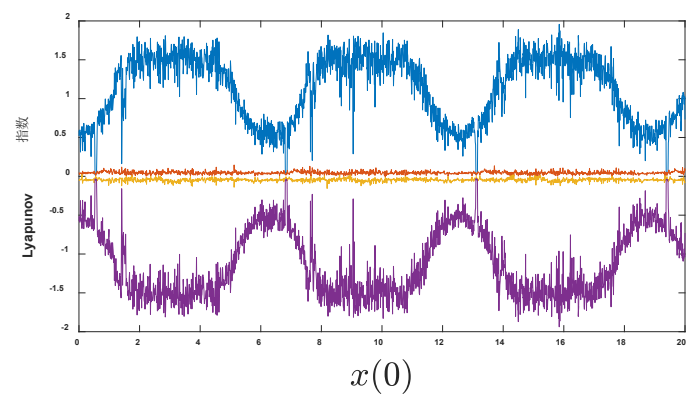

Fig. 1. Lyapunov exponent diagram

It can be seen from Fig. 1 that the Lyapunov exponent of the system (5) is symmetric about the $x$-axis, and the maximum exponent is always greater than 0 , so the system is always in a chaotic state. Secondly, the Lyapunov exponent of the system shows a periodic distribution of $2 \pi$.

\subsection{Multi-stability of the system (5)}

(C) The 2021 International Conference on Artificial Life and Robotics (ICAROB2021), January 21 to 24,2021 


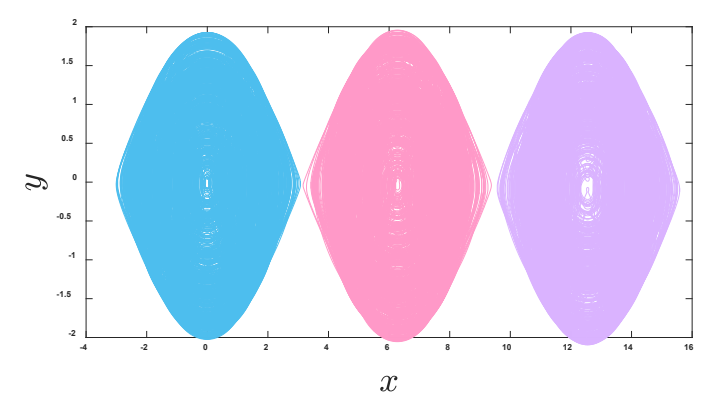

Fig. 2. Coexistence of three independent scrolls

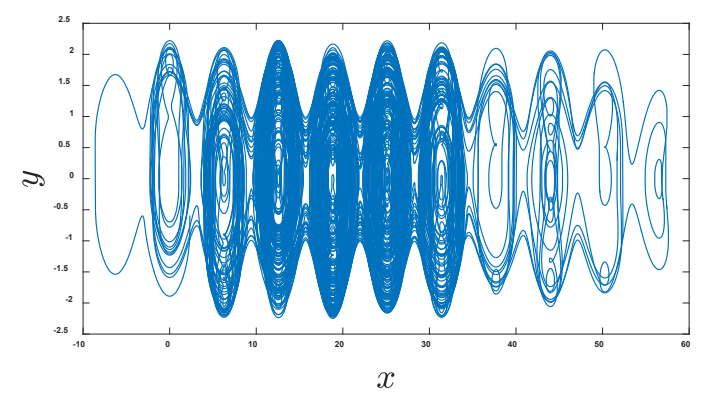

(a) 11-scrolls

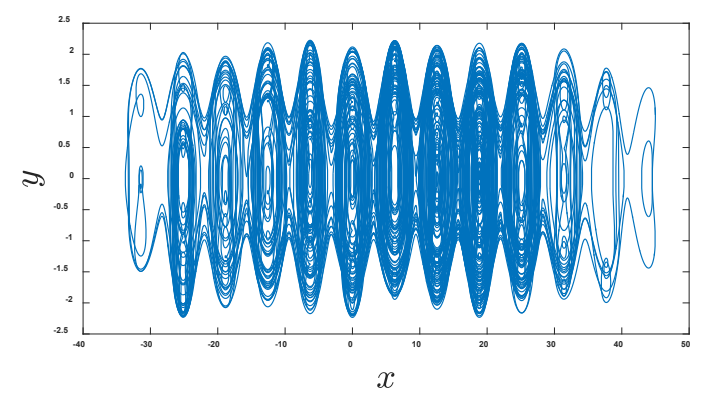

(b) 13-scrolls

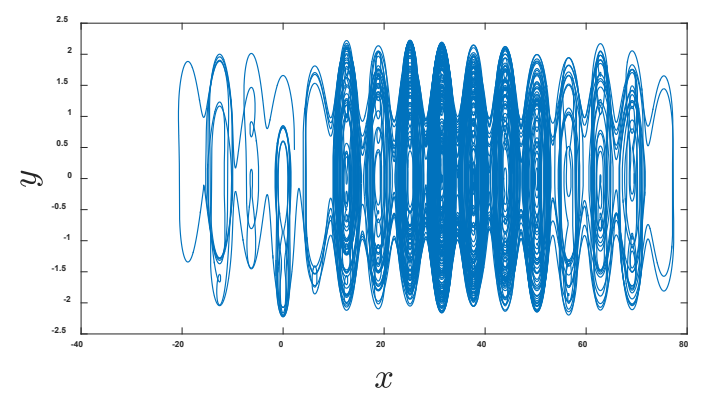

(c) 16-scrolls

Fig. 2. Different numbers of multi-scroll flows with the change of initial value

It can be found that different flows of the system (5) will appear with the change of initial value, which are shown in Fig. 2 and Fig. 3. When the initial value is $\left(\frac{\pi}{3}+\right.$ $2 k \pi, 1,1,1)(k=0,1,2)$, there are three independent scroll flows in the system (5), which are represented by blue, pink and purple lines in Fig. 2. When the initial value is $\left(\frac{\pi}{2}+2 k \pi, 1,1,1\right)(k=0,1,2)$, there are 11 -scrolls flow, 13-scrolls flow and 16-scrolls flow in the system (5), which are represented by blue, pink and purple lines in Fig. 2. According to the above analysis, it can be seen that the coexistence of different multi-scrolls flows with the change of different initial values illustrates the multistability of the system (5).

\subsection{Grid type multi-scroll flows}

In the above, multi-scroll flows are obtained in the system (5) in the $\mathrm{x}$-axis direction by expanding the equilibrium point in the $\mathrm{x}$-axis direction. Similarly, the construction of the grid type multi-scroll flows needs to expand the equilibrium point in the $\mathrm{x}$-axis and $\mathrm{Y}$-axis directions. Set $\nabla H=(f(x), f(y), z, w)$, and equation (2) can be express as:

$$
\left\{\begin{array}{l}
\dot{x}=4 f(y)+4 f(y) w \\
\dot{y}=-4 f(x) \\
\dot{z}=4 w \\
\dot{w}=-4 f(x) f(y)-4 z
\end{array}\right.
$$

Where $f(x)$ and $f(y)$ satisfy mapping:

$$
f(u)=\left\{\begin{array}{c}
u+N, \quad u<-N \\
\sin (u),-N \leq u \leq N, N=2 n \pi, n \in Z^{*} \\
u-N, \quad u>N
\end{array}\right.
$$

It can be found that the equilibrium point of the system is $(k \pi, k \pi, 0,0), k \in Z^{*}$. When the equilibrium point is $(2 k \pi, 2 k \pi, 0,0)$ and $((2 k-1) \pi,(2 k-1) \pi, 0,0)$, the corresponding eigenvalue is $(4 i,-4 i, 4 i,-4 i)$ and the equilibrium point type is a center point. When the equilibrium point is $((2 k-1) \pi, 2 k \pi, 0,0)$ and $(2 k \pi,((2 k-1) \pi, 0,0)$, the corresponding eigenvalue is $(4,-4,4 i,-4 i)$ and the type of the equilibrium point is an unstable saddle point.

In addition, the scroll of the system (6) will be generated at the center point, and the bond band will be generated at the unstable saddle point. When $N=2 n \pi$, the equilibrium point of the system (6) has $(2 n)^{2}+(2 n+$ $1)^{2}$ central points and $4 n \times(2 n+1)$ unstable saddle points. Then, a multi-scroll flow with $(4 n+1) \times(4 n+$ 
1) grid type is formed, it is composed of $(2 n)^{2}+$ $(2 n+1)^{2}$ vortices and $4 n \times(2 n+1)$ bond bands.

Set $n=1,2,5 \times 5$ and $9 \times 9$ grid type multi-scroll flows are shown in Fig. 3.

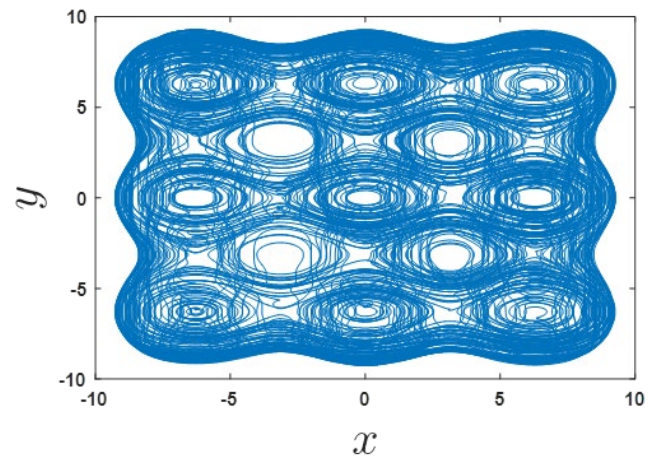

(a) $5 \times 5$ grid type multi-scroll flow

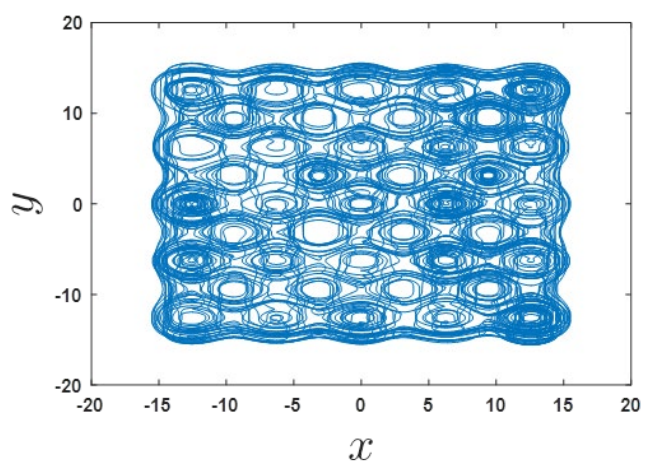

(b) $9 \times 9$ grid type multi-scroll flow

Fig. 3. grid type multi-scroll flows

\subsection{NIST test of system (6)}

At present, NIST SP800-22 standard is the most widely used and authoritative standard used to detect the pseudorandom sequence generated by the system (6). It has 15 test indexes. The ideal random sequence is taken as a reference to test the deviation degree of pseudo-random sequence from different angles in statistical characteristics. It is generally believed that the sequence that can pass the detection has a good pseudo-random performance. Each test of SP800-22 standard will provide the pass rate and the uniformity of $\mathrm{P}$ value distribution. If the significance level $\alpha=0.01$ and the test sequence is group $\beta$, the confidence interval of the passing rate can be defined as:
The test conditions used in this paper are: significance level $\mathrm{a}=0.01$, test sequence $\mathrm{B}=100$, length of each group is $10 \mathrm{bit}$, and the confidence interval is 1 . The test results are shown in Fig. 4.

\begin{tabular}{cccccc}
\hline No. & Statistical Test & P-value & Test Times & proportion & Result \\
\hline 1 & Frequency & 0.867692 & 1 & 0.99 & Pass \\
2 & Block Frequency & 0.319084 & 1.00 & 1.00 & Pass \\
3 & Cumulative Sums & 0.236810 & 2 & 0.99 & Pass \\
4 & Runs & 0.678686 & 1 & 1.00 & Pass \\
5 & Longest Run & 0.897763 & 1 & 0.97 & Pass \\
6 & Rank & 0.924076 & 1 & 0.99 & Pass \\
7 & FFT & 0.236810 & 1 & 0.99 & Pass \\
8 & NonOverlapping Template & 0.017912 & 148 & 0.97 & Pass \\
9 & Overlapping Template & 0.474986 & 1 & 0.98 & Pass \\
10 & Universal & 0.137282 & 1 & 1.00 & Pass \\
11 & Approximate Entropy & 0.657933 & 1 & 1.00 & Pass \\
12 & Random Excursions & 0.051391 & 8 & 1.00 & Pass \\
13 & Random Excursions Variant & 0.010606 & 18 & 0.97 & Pass \\
14 & Serial & 0.350485 & 2 & 1.00 & Pass \\
15 & Linear Complexity & 0.062821 & 0.99 & 1.00 & Pass \\
\hline
\end{tabular}

Fig. 4. The results of NIST test

The performance of pseudo-random sequence is comprehensively analyzed in the experiment. The experiment can pass only when the following conditions are met:

- All P-values must be greater than significance level $\alpha$.

- The pass rate must be in the confidence interval.

- P-values must be evenly distributed.

It can be seen from Fig. 4 that the system (6) has passed the test and proved that the system has good pseudo randomness.

In this paper, taking Non-Overlapping Template as an example, the system (6) can be found to satisfy the uniform distribution of P-value. P-value distribution of NonOverlapping Template is shown in Fig.5.

$$
\left(1-\alpha-3 \sqrt{\frac{\alpha(1-\alpha)}{\beta}}, 1+\alpha-3 \sqrt{\frac{\alpha(1-\alpha)}{\beta}}\right)
$$




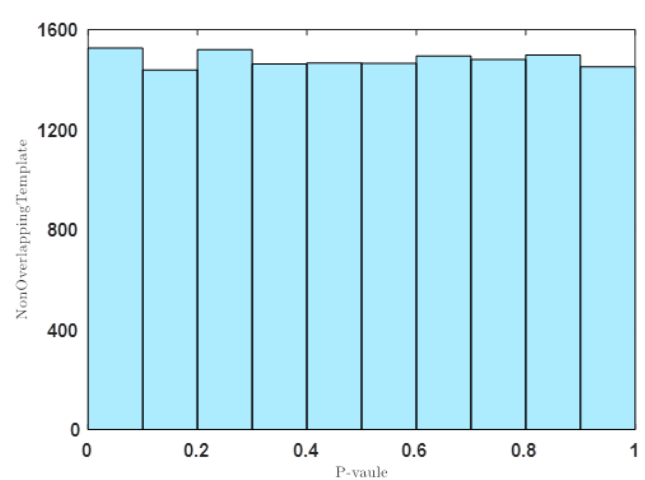

Fig. 5. P-value distribution of Non-Overlapping Template

\section{Conclusion}

In this paper, a four-dimensional conservative system with a unique equilibrium point is constructed. Firstly, the sine function is introduced to extend the equilibrium point in the $\mathrm{x}$-axis direction so that it has a linear equilibrium point with infinite extension in the x-axis direction. Secondly, it is found that the system (5) has different numbers of multiscroll flows under different initial values, which proves that the system has multi-stability. Thirdly, by introducing a sinusoidal function to extend the equilibrium points in the $\mathrm{x}$-axis and $\mathrm{Y}$-axis directions, a controllable grid type multi-scroll flow is found. Finally, the system is tested by NIST, and the test results show that the system has good pseudo randomness.

\section{Acknowledgements}

\section{References}

1. 1. Suykens, J.A.K., Vandewalle, J., Generation of n-double scrolls $(n=1,2,3,4, \ldots)$, IEEE $T$ rans. Circuits Syst. IFundam. Theory Appl, 1993, 40, 861-867.

2. Zhang, C.X.; Yu, S.M. Design and implementation of a novel multi-scroll chaotic system. Chin. Phys. B 2009, 18, 119-129.

3. Wang, F.Q.; Liu, C.X. A new multi-scroll chaotic generator. Chin. Phys. 2007, 16, 942-945.

4. Liu, X.Z.; Shen, X.M.; Zhang, H.T. Multi-scroll chaotic and hyperchaotic attractors generated from Chen system. Int. J. Bifurc. Chaos 2012, 22, 1250033.

5. Chen, D.Y; Sun, Z.T.; Ma, X.Y .; Chen, L. Circuit implementation and model of a new multi-scroll chaotic system. Int. J. Circuit Theory Appl. 2014, 42, 407-424.
6. Luo, X.H.; Tu, Z.W.; Liu, X.R.; Cai, C.; Liang, Y .L.; Gong, P . Implementation of a novel two-attractor grid multi-scroll chaotic system. Chin. Phys. B 2010, 19, 070510.

7. Chen, Z.; Wen, G.L.; Zhou, H.A.; Chen, J.Y . A new M x Ngrid double-scroll chaotic attractors from Rucklidge chaotic system. Optik 2017, 136, 27-35.

8. Chen, Z.; Wen, G.L.; Zhou, H.A.; Chen, J.Y . A new M x Ngrid double-scroll chaotic attractors from Rucklidge chaotic system. Optik 2017, 136, 27-35.

9. Lü, J.H.; Han, F.L.; Yu, X.H.; Chen, G.R. Generating 3-D multiscroll chaotic attractors: A hysteresis series switching method. Automatica 2004, 40, 1677-1687.

10. Peng, Z.P .; Wang, C.H.; Luo, X.W. A novel multi-directional multi-scroll chaotic system and its CCll+ circuit implementation. Optik 2014, 125, 6665-6671.

11. Wang, C.H.; Xu, H.; Yu, F. A novel approach for constructing high-order Chua's circuit with multi-directional multi-scroll chaotic attractors. Int. J. Bifurc. Chaos 2013, 23, 13500223.

12. Deng, W.H.; Lü, J.H. Generating multi-directional multiscroll chaotic attractors via a fractional differential hysteresis system. Phys. Lett. A 2007, 369, 438-443.

13. Zhang, Y .; Yu, S.M.; Liu, M.H. Generating multi-scrol hyperchaotic attractors based on FPGA technology. Circuits Syst. 2007, 12, 39-43.

14. Tlelo-Cuautle, E.; Rangel-Magdaleno, J.J.; Pano-Azucena, A.D.; Obeso-Rodelo, P .J.; Nunez-Perez, J.C. FPGA realization of multi-scroll chaotic oscillators. Commun. Nonlinear Sci. Numer. Simul. 2015, 27, 66-80.

15. Yu, S.M.; Lü, J.H.; Leung, H.; Chen, G.R. Design and implementation of $n$-scroll chaotic attractors from a general jerk circuit. IEEE T rans. Circuits Syst. I Regul. Pap. 2005, 52, 1459-1476.

16. Yalcin, M.E. Multi-scroll and hypercube attractors from a general jerk circuit using Josephson junctions. Chaos Solitons Fractals 2007, 34, 1659-1666.

17. He, S.B.; Sun, K.H.; Wang, H.H.; Ai, X.X.; Xu, Y .X. Design of n-dimensional multi-scroll Jerk chaotic system and its performance. J. Appl. Anal. Comput. 2016, 6, 1180-1194.

18. Ma, J.; Wu, X.Y .; Chu, R.T.; Zhang, L.P. Selection of multiscroll attractors in Jerk circuits and their verification using Pspice. Nonlinear Dyn. 2014, 76, 1951-1962.

19. T chitnga, R.; Nguazon, T.; Fotso, P .H.L.; Gallas, J.A.C. Chaos in a Single Op-Amp-Based Jerk Circuit: Experiments and Simulations. IEEE T rans. Circuits Syst. II Express Briefs 2016, 63, 239-243.

20. Tang, W.K.S.; Zhong, G.Q.; Chen, G.; Man, K.F. Generation of $n$-scroll attractors via sine function. IEEE $T$ rans. Circuits Syst. I Fundam. Theory Appl. 2001, 48, 1369-1372.

21. Hu, X.; Liu, C.; Liu, L.; Ni, J.K.; Li, S.L. Multi-scroll hidden attractors in improved Sprott A system. Nonlinear Dyn. 2016, 86, 1725-1734.

22. Luo, X.H. Circuitry implementation of a novel nonautonomous hyperchaotic Liu system based on sine input. Chin. Phys. B 2009, 18, 3304-3308. 